\title{
Filter Bank Based Fractional Delay Filter Implementation for Widely Accurate Broadband Steering Vectors
}

\author{
Mohamed A. Alrmah and Stephan Weiss \\ Department of Electronic \& Electrical Engineering, University of Strathclyde,Glasgow,Scotland, UK \\ \{mohamed.alrmah;stephan.weiss\}@ strath.ac.uk
}

\begin{abstract}
Applications such as broadband angle of arrival estimation require the implementation of accurate broadband steering vectors, which generally rely on fractional delay filter designs. These designs commonly exhibit a rapidly decreasing performance as the Nyquist rate is approached. To overcome this, we propose a filter bank based approach, where standard fractional delay filters operate on a series of frequency-shifted oversampled subband signals, such that they appear in the filter's lowpass region. Simulations demonstrate the appeal of this approach.
\end{abstract}

\section{INTRODUCTION}

In broadband sensor array signal processing, time delays that arise from wave fronts propagating across the array at finite speed require to be addressed as lags rather than be represented by phase shifts as in the narrowband case. Normally non-integer multiples of the sampling period, fractional delay filters need to be employed [1], [2]. Since sensor array applications potentially operate across several octaves, the accuracy of such fractional delays is crucial to the precision of broadband angle of arrival estimation or the performance of any other subsequent processing [3].

To implement fractional delays, a sinc function can be appropriately sampled [4], [5] and restricted to finite support by truncation. This leads to discrete prolate spheroidal sequences [6], which suffer from a ripple in the group delay and a degradation in performance that increases with frequency. This restricted accuracy of fractional delay filters [5] often limits their application to lowpass-type signals.

In order to enhance the fractional delay filters' performance, tapered instead of rectangular windows have been proposed to truncate the sinc function [4], [7], leading to a lower group delay ripple. A polynomial approximation approach was introduced by Farrow [8], which, at a modest filter order, provides relatively good accuracy. However, both Farrow structure and windowed sinc still perform best a low frequencies, with a significant performance degradation towards higher frequencies.

Subbands created by decimated filter banks have previously been used in the context of fractional delays, since decimation can shorten long impulse responses such as sinc functions [9]. Recognising that recent fractional delay approaches are fairly accurate at low frequencies and only degrade towards higher frequencies, this paper proposes to modulate undecimated subbands as created by filter banks to acquire lowpass characteristics. After applying fractional delay filters in the lowpass domain, a frequency shift to the original band is performed, and a synthesis filter bank operation completes the proposed accurate broadband fractional delay filter approach.

Below, Sec. II motivates the requirement of highly accurate fractional delay filters by reviewing the construction of broadband steering vectors. Sec. III reviews different approaches for designing fractional delay filters followed by the proposed filter bank approach outlined in Sec. IV. Simulation results are presented in Sec. V to compare and characterise the accuracy of the proposed approach to various benchmarks. Conclusions are drawn in Sec. VI.

\section{Broadband Steering Vectors}

An $M$-element array of omnidirectional sensors located at positions $\mathbf{r}_{m}, m=1 \ldots M$ collects a signal vector $\mathbf{x}(t) \in \mathbb{C}^{M}$, with the continuous time variable $t$. If a far field source illuminates the array such that the signal at the origin $\mathbf{r}=\underline{0}$ is $s(t)$ and we neglect attenuation, then

$$
\mathbf{x}(t)=\left[\begin{array}{c}
s\left(t-T_{1}\right) \\
s\left(t-T_{2}\right) \\
\vdots \\
s\left(t-T_{M}\right)
\end{array}\right]=\left[\begin{array}{c}
\delta\left(t-T_{1}\right) \\
\delta\left(t-T_{2}\right) \\
\vdots \\
\delta\left(t-T_{M}\right)
\end{array}\right] * s(t)
$$

with $*$ denoting convolution, and delays $T_{m}=\frac{1}{c} \mathbf{k}^{\mathrm{T}} \mathbf{r}_{m}, m=$ $1 \ldots M$, where $\mathbf{k}$ is the normal vector of the source's wave front, and $\mathbf{k} / c$ is known as the slowness vector of the source.

Sampling $\mathbf{x}(t)$ with a period $T_{s}$ yields $\mathbf{x}[n]$, with discrete time index $n$ such that $t=n T_{s}$. Under the assumption of a perfectly bandlimited signal $s(t)$, the interpolator underlying the sampling process is a sinc function. With

$$
\mathbf{x}[n]=\left[\begin{array}{c}
\delta\left[n-\tau_{1}\right] \\
\delta\left[n-\tau_{2}\right] \\
\vdots \\
\delta\left[n-\tau_{M}\right]
\end{array}\right] * s[n]=\mathbf{a}[n] * s[n]
$$

and normalised delays $\tau_{m}=T_{m} / T_{s}$, the ideal fractional delays $\delta\left[n-\tau_{m}\right]$,

$$
\delta[n-\tau]=\left\{\begin{array}{lll}
\frac{\sin (\pi(n-\tau))}{\pi(n-\tau)} & , & n \neq \tau \\
1 & , & n=\tau
\end{array}\right.
$$


are now sinc functions which not necessarily remain sampled in the sinc's zero crossing, and therefore generally possess infinite support. The quantity $\mathbf{a}[n]$ in (2) is referred to as broadband steering vector, and consists of a number of different fractional delays of the type in (3).

A signal model for a scenario with $L$ independent far field broadband sources $s_{l}[n], l=1 \ldots L$, each characterised by a broadband steering vector $\mathbf{a}_{l}[n]$, therefore becomes

$$
\mathbf{x}[n]=\sum_{l=1}^{L} \sum_{v=0}^{\infty} \mathbf{a}_{l}[v] s_{l}[n-v]+\mathbf{v}[n],
$$

with $\mathbf{v}[n]$ representing spatially and temporally uncorrelated noise with covariance $\mathscr{E}\left\{\mathbf{v}[n] \mathbf{v}^{\mathrm{H}}[n]\right\}=\sigma_{v}^{2} \mathbf{I}$. To capture information contained in the data vector $\mathbf{x}[n]$ requires a spacetime covariance matrix $\mathbf{R}[v]=\mathscr{E}\left\{\mathbf{x}[n] \mathbf{x}^{\mathrm{H}}[n-v]\right\}$ with lag parameter $v$. Its Fourier pair, the cross-spectral density matrix $\mathbf{R}(z)=\sum_{v} \mathbf{R}[v] z^{-v}$ or short $\mathbf{R}[v] \circ-\bullet \mathbf{R}(z)$,

$$
\mathbf{R}(z)=\sum_{l=1}^{L} \mathbf{a}(z) \mathbf{a}^{\mathrm{H}}\left(z^{-1}\right) R_{l}(z)+\sigma_{v}^{2} \mathbf{I}
$$

with $R_{l}(z)$ the power spectral density of the $l$ th source, forms a polynomial matrix.

A number of broadband array methods directly utilise the broadband steering vector. For example, in [3] broadband steering vectors are used to presteer array data. The parametric covariance matrix approach in [2], [10] presteers the data prior to scanning for maximised eigenvalues in the resulting covariance matrix. For the polynomial MUSIC algorithm in [1], a polynomial eigenvalue decomposition [11] of the space-time covariance matrix in (5) identifies the noise-only subspace, which can then be scanned using broadband steering vectors, in analogy to the narrowband MUSIC algorithm [12]. Thus, the accuracy of the broadband steering vector implementation impacts crucially on all of these applications.

\section{Fractional Delay Filters}

With the definition of the ideal fractional delay and an error metric defined in Sec. III-A, this section reviews various implementation methods for fractional delay filters, including windowed sinc functions in Sec. III-B and the Farrow structure [8] in Sec. III-C.

\section{A. Ideal Delay and Performance Metric}

Based on the definition of the ideal fractional delay in (3),

$$
f_{\text {ideal }}[n]=\delta[n-\tau] \quad,
$$

and the Fourier pair $\delta[n] \circ-\bullet 1$, the Fourier transform of the fractional delay yields

$$
F_{\text {ideal }}\left(e^{j \Omega}\right)=1 \cdot e^{-j \Omega \tau}
$$

with a group delay $\gamma_{\text {ideal }}=\tau$. Using this ideal delay, an error metric for an arbitrary fractional delay filter approximation $f[n]$ can be defined as

$$
S_{e e}\left(e^{j \Omega}\right)=\left|F_{\text {ideal }}\left(e^{j \Omega}\right)-F\left(e^{j \Omega}\right)\right|^{2} \quad,
$$

with $F\left(e^{j \Omega}\right) \bullet-\circ f[n]$, such that $S_{e e}\left(e^{j \Omega}\right)$ is a quadratic error metric for the approximation of $f_{\text {ideal }}[n]$ by $f[n]$.

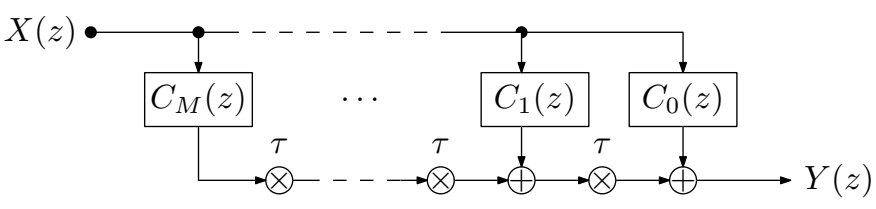

Fig. 1: Farrow structure with $M+1$ subsystems of order $L$ approximating a fractional delay $\tau$ between input $X(z)$ and output $Y(z)$.

\section{B. Windowed Sinc Methods}

Since the ideal fractional delay in (6) possesses infinite support, in general a window $w_{N}[n]$ and time delay is applied to create a causal filter of length $2 N$,

$$
f[n]=f_{\text {ideal }}[n-\tau-N] w_{N}[n-\tau-N] \quad .
$$

In the simplest case, a rectangular window $w_{N}[n]=p_{N}[n]$ performs a truncation according to

$$
p_{N}[n]=\left\{\begin{array}{lll}
1 & , & |n| \leq N \\
0 & , & |n|>N
\end{array} .\right.
$$

The resulting discrete prolate spheroidal sequence $f[n]$ provides an approximation of $f_{\text {ideal }}[n]$ that generally improves with $N$ at lower frequencies. However, independent from $N$, the performance degrades due Gibbs phenomena as the Nyquist frequency is approached [13].

The approximation of an ideal fractional delay can be improved by introducing a tapered winod [4], [7], such as a Hann window

$$
w_{N, \operatorname{Hann}}[n]=\cos ^{2}\left(\frac{\pi n}{2 N}\right) p_{N}[n]
$$

Such windowing techniques can reduce the ripple in the frequency response, lowering the error metric in (8) at lower frequencies.

\section{Farrow Structure}

The Farrow structure [8] implements a polynomial interpolation between input samples. Consisting of $M+1$ sections of $L$ th-order FIR filters $C_{m}(z), m=0 \ldots M$, which provide an interpolation between input samples, Fig. 1 shows the diagram of the Farrow structure. The fractional delay of the structure is given by the single fractional delay parameter $\tau$, leading to a transfer function

$$
F(z)=\sum_{m=0}^{M} C_{m}(z) \tau^{m}
$$

For a given fractional delay $\tau, F(z)$ is fixed. The magnitude response of the Farrow structure is flat at low frequencies only, thus limiting its applicability to broadband problems that extend beyond lowpass-type signals.

\section{Filter Bank Approach}

Noting tha various fractional delay filters reviewed in Sec. III perform best at low frequencies, the filter bank based structure in Fig. 2 is proposed as an implementation framework for fractional delay filters. In this structure, the input signal is 


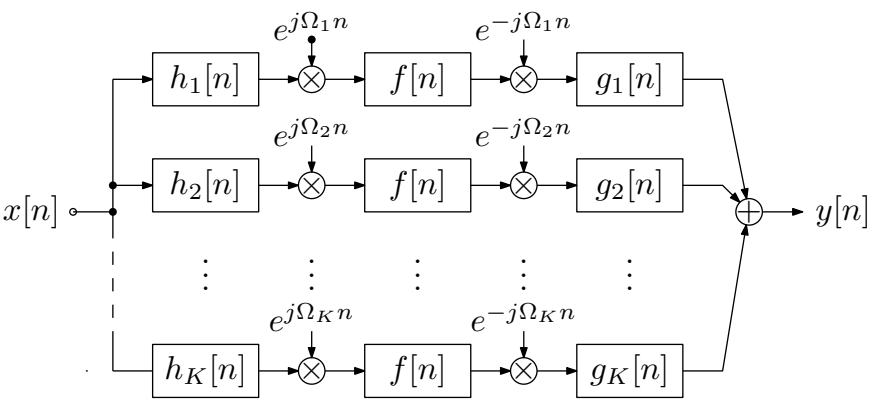

Fig. 2: Proposed subband-based fractional delay filter with analysis filter bank stage with analysis filters $h_{k}[n] \circ-\bullet H_{k}(z)$, a modulation stage, the fractional delay filters $f[n]$, a demodulation stage, followed by a synthesis filter bank with synthesis filters $g_{k}[n] \circ \longrightarrow G_{k}(z)$.

split into $K$ different frequency bands using an analysis filter bank with filters $H_{k}(z), k=1 \ldots K$. Undecimated, the subband signals are frequency shifted by $\Omega_{k}$ such that the fractional delay filters are applied to lowpass signals in every branch. After fractionally delaying the subband signals, the frequency shifts are reversed, and signal are combined using a suitable bank of synthesis filters $G_{k}(z)$.

To reduce memory and computational requirements, the analysis filter can be derived from a common lowpass prototype filter by means of a modulating transform. We here use generalised discrete Fourier transform (GDFT) modulated filter banks, which offer advantages over other modulations in terms of subband uniformity and the ability to implement a near-perfect paraunitary system, where the synthesis filters $G_{k}(z)$ can be derived by time reversal from the analysis filters [14]. The prototype filter can be designed using a leastsquares approach [14], whereby the reconstruction error of the filter bank is a design criterion that is optimised. Therefore, depending on the quality of the prototype filter, and therefore its length and complexity, different levels of reconstruction errors can be achieved for the filter bank.

A sample filter bank characteristic with $K=16$ subbands is shown in Fig. 3. The bandpass nature of the subband signals motivates the modulation by

$$
\Omega_{k}=\frac{(2 k-1) \pi}{K} \quad k \in \mathbb{Z}, k=1 \cdots K,
$$

which translates every subband in frequency to sit symmetrically around $\Omega=0$.

\section{Simulations and Numerical Results}

Fig. 4 shows the approximation error for a truncated sinc function with $N=100$, where a maximum error is reached for a fractional delay of $\tau=\frac{1}{2}$ and frequencies approaching the Nyquist rate. The degradation towards the Nyquist rate is shared by the Hann-windowed sinc function in Fig. 5, also with window $N=100$, and a Farrow structure with polynomial order $M=3$ in Fig. 6. While the Farrow structure for a low polynomial degree does not perform well for higher

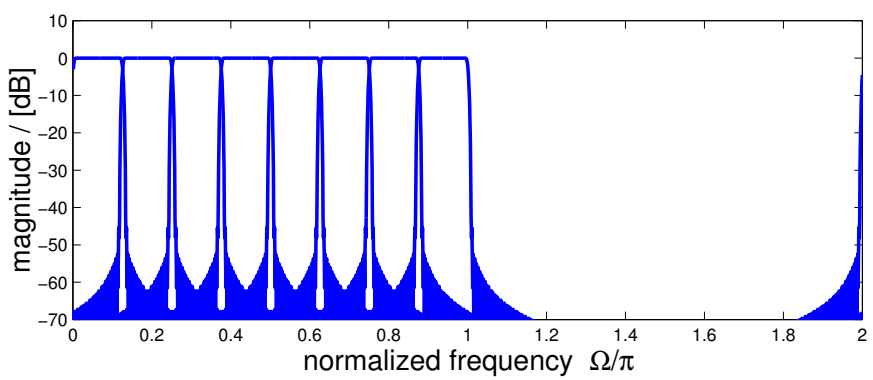

Fig. 3: Characteristic of the lower $K / 2$ analysis filters $H_{k}\left(e^{j \Omega}\right) \bullet-\circ h_{k}[n]$ of a $K$ channel filter bank.

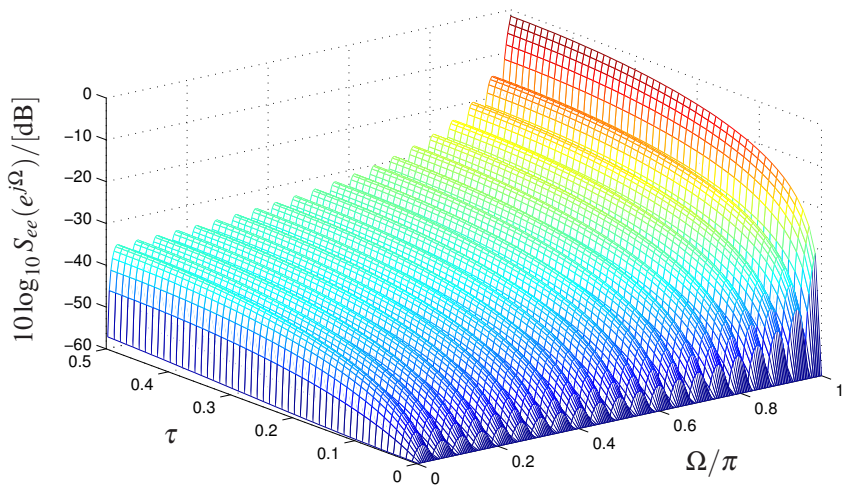

Fig. 4: Approximation error for truncated sinc function with $N=100$.

frequencies, it significantly exceeds both the rectangular and Hann-windowed sinc approach, whereby the Hann-window offers advantages over the rectangular window at almost no cost.

For the proposed filter bank approach to a fractional delay implementation, Fig. 7 shows the combination of a $K=16$ channel filter bank with an $M=3$ order Farrow structure to implement the fractional delay in the frequency-shifted subbands. As the results in Fig. 7, the error is uniformly low

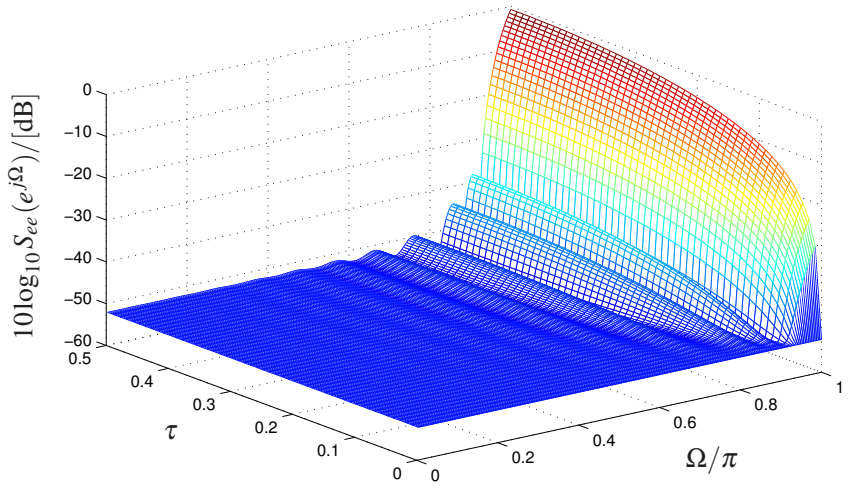

Fig. 5: Approximation error for Hann windowed sinc function with $N=100$. 


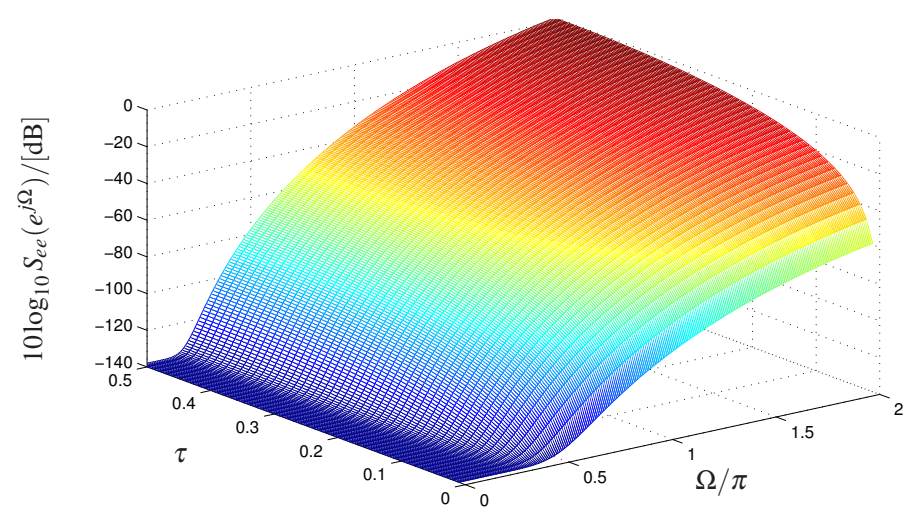

Fig. 6: Approximation error for Farrow structure for $M=3$.

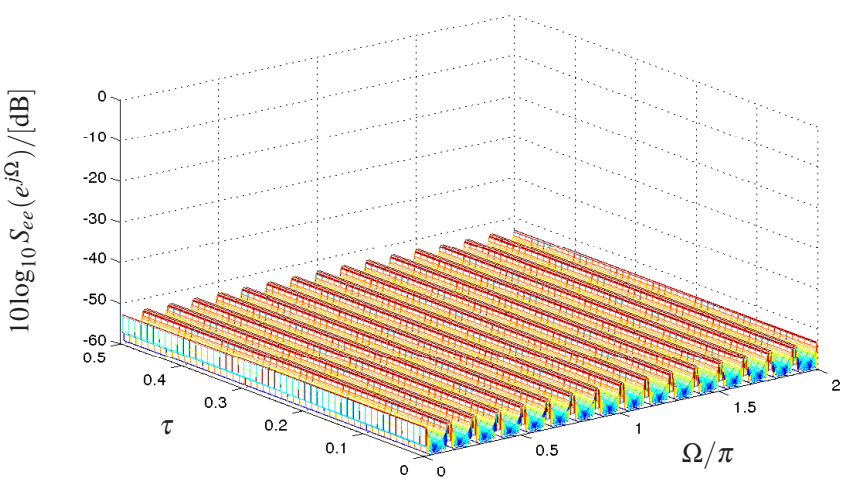

Fig. 7: Approximation error for filter bank approach with with $K=16$, and an $L=3$ order Farrow filter as subband fractional delay $f[n]$.

\section{REFERENCES}

[1] M. Alrmah, S. Weiss, and S. Lambotharan, "An extension of the music algorithm to broadband scenarios using polynomial eigenvalue decomposition," in 19th European Signal Processing Conference, Barcelona, Spain, August 2011, pp. 629-633.

[2] J. Dmochowski, J. Benesty, and S. Affes, "Direction of arrival estimation using the parameterized spatial correlation matrix," IEEE Transactions on Audio, Speech, and Language Processing, vol. 15, no. 4, pp. 13271339, May 2007.

[3] P. Murphy, A. Krukowski, and A. Tarczynski, "An efficient fractional sample delayer for digital beam steering," in IEEE International Conference on Acoustics, Speech, and Signal Processing, vol. 3, Munich, Germany, April 1997, pp. 2245-2248.

[4] A. Yardim, G. Cain, and P. Henry, "Optimal two-term offset windowing for fractional delay," Electronics Letters, vol. 32, no. 6, pp. 526-527, March 1996.

[5] T. I. Laakso, V. Välimäki, M. Karjalainen, and U. K. Laine, "Splitting the Unit Delay," IEEE Signal Processing Magazine, vol. 13, no. 1, pp. 30-60, January 1996.

[6] A. Papoulis, Signal Analysis. New York: McGraw-Hill, 1984.

[7] J. Selva, "An efficient structure for the design of variable fractional delay filters based on the windowing method," IEEE Transactions on Signal Processing, vol. 56, no. 8, pp. 3770-3775, August 2008.

[8] C. W. Farrow, "A continuously variable digital delay element," in IEEE International Symposium on Circuits and Systems, vol. 3, Espoo, FInland, June 1988, pp. 2641-2645.

[9] S. Weiss, S. R. Dooley, R. W. Stewart, and A. K. Nandi, "Adaptive Equalisation in Oversampled Subbands," IEE Electronics Letters, vol. 34, no. 15, pp. 1452-1453, July 1998.

Accurate broadband steering vector requirements for applications such as broadband angle of arrival estimation have motivated the implementation of fractional delay filters that can approach the ideal fractional delay over a large bandwidth. Since state-of-the-art fractional delays such as windowed sinc and Farrow filters perform best at low frequencies only, we have combined these filters with a modified filter bank, whereby undecimated subband signals are modulated such that only a small lowpass region is active in each subband. The subband signals can then be accurately delayed by any of the established methods.

As demonstrated in simulations, uniform accuracy can be achieved across the entire bandwidth, whereby the approximation error w.r.t. an ideal delay is either limited by the fractional delay filter or the reconstruction error of the filter bank.
[10] M. Souden, J. Benesty, and S. Affes, "Broadband source localization from an eigenanalysis perspective," IEEE Transactions on Audio, Speech, and Language Processing, vol. 18, no. 6, pp. 1575-1587, August 2010.

[11] J. G. McWhirter, P. D. Baxter, T. Cooper, S. Redif, and J. Foster, "An EVD Algorithm for Para-Hermitian Polynomial Matrices," IEEE Transactions on Signal Processing, vol. 55, no. 5, pp. 2158-2169, May 2007.

[12] R. O. Schmidt, "Multiple emitter location and signal parameter estimation," IEEE Transactions on Antennas and Propagation, vol. 34, no. 3, pp. 276-280, March 1986.

[13] M. Sac and M. Blok, "Gain deficit effect in the fractional delay filter design by the window method," in Proc. SPIE Photonics Applications in Astronomy, Communications, Industry, and High-Energy Physics Experiments, vol. 75021G, August 2009, pp. 1-6.

[14] M. Harteneck, S. Weiss, and R. Stewart, "Design of near perfect reconstruction oversampled filter banks for subband adaptive filters," IEEE Transactions on Circuits and Systems II: Analog and Digital Signal Processing, vol. 46, no. 8, pp. 1081-1085, August 1999. 